\title{
What's Positive and Negative about Generics: \\ A Constrained Indexical Approach
}

Junhyo Lee

Anthony Nguyen

\section{Introduction}

Nguyen (2020) introduced the positive data and argued that only his pragmatic account and Sterken's (2015a) indexical account can accommodate it. In this paper, we will present new data - what we call the negative data - and argue that there is no theory on the market that is compatible with both the positive data and the negative data. We will draw two generalizations from them and develop a novel version of the indexical account that captures both the positive data and the negative data. In particular, we argue that there is a semantic constraint that, in any context, the semantic value of GEN is upward monotone and nonsymmetric. Whereas the indexical account can accommodate these data when appropriately modified, the pragmatic account cannot. This is because no pragmatic principles have been developed that can explain the negative data. While we focus on only the pragmatic account and the indexical account, the data and generalizations discussed here are of broad interest, as they must be accommodated by any empirically adequate account of generics. 


\section{The Positive Data and Its Implication}

Generics, which are often expressed using bare plurals (e.g., "Tigers are striped"), have received much attention from both philosophers and linguists. ${ }^{1}$ But generics have proved resistant to systematic semantic theorizing. This is mainly because generics can be used to express propositions with radically different quantificational forces: that of universal quantifiers (e.g., “all”), modalized universal quantifiers (e.g., "all ... can," "ideally, all," "under normal circumstances, all"), proportional quantifiers (e.g., "most," "many"), and quasi-existential quantifiers (e.g., "a few”). Nguyen (2020) calls this data The Variety Data, but we prefer to call it The Positive Data: ${ }^{2}$

\section{The Positive Data (The Variety Data) ${ }^{3,4}$}

\section{(1) $\left[\right.$ A few] mosquitoes transmit malaria. ${ }^{5}$}

\footnotetext{
${ }^{1}$ For broad introductions to the literature on generics, see Krifka et al. (1995), Leslie (2016), and Sterken (2017).

${ }^{2}$ In the absence of an explicitly supplied context, we will assume typical contexts. Bach (2002) and Bach (2005) have argued that in the absence of an explicitly provided context, speakers evaluate sentences by imagining a typical one. Some evidence that speakers can automatically supply a typical context is the felicity of some sentences containing context-dependent expressions despite the absence of any explicitly offered context. For example, "She left Bob" can be understood as meaning "Bob's female ex-partner left Bob" despite the lack of any explicitly offered context to help resolve the reference of "she."

${ }^{3}$ Only the unbracketed material is overtly pronounced. The bracketed material indicates the quantificational force of what is asserted.

${ }^{4}$ Here are sample lexical entries for the quantifiers in the positive data:

$\llbracket “$ “all” $\rrbracket^{\mathrm{c}}=\lambda \mathrm{F} \cdot \lambda \mathrm{G} \cdot \frac{\#(\mathrm{~F} \& \mathrm{G})}{\# \mathrm{~F}}=1$

$\llbracket$ “almost all” $\rrbracket^{\mathrm{c}}=\lambda \mathrm{F} . \lambda \mathrm{G} . \frac{\#(\mathrm{~F} \& \mathrm{G})}{\# \mathrm{~F}} \geq \mathrm{n}$, where $\mathrm{n}$ is some number very close to 1 (e.g., 0.9 ).

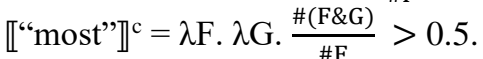

$\llbracket “$ many" $\rrbracket^{\mathrm{c}}=\lambda \mathrm{F} . \lambda \mathrm{G} . \frac{\#(\mathrm{~F} \& \mathrm{G})}{\# \mathrm{~F}} \geq 0.3$.

$\llbracket “ a$ few" $\rrbracket^{c}=\lambda F \cdot \lambda G . \frac{\#(F \& G)}{\# F} \geq n$, where $n$ is some number very close to 0 (e.g., 0.01 ).

${ }^{5}$ The proportional reading of "a few", not its cardinal reading, is intended.
} 
(2) [Many] barns are red.

(3) [All] prime numbers are odd.

(4) [Under normal circumstances, almost all] ravens are black.

(5) [By definition, all] round squares are round.

(6) [Ideally, all] boys don't cry.

(7) [Most] orange crushers [can] crush oranges.

Nguyen (2020) argues that only his account and Sterken's (2015a) can accommodate this data. Intuitively, the problem with other accounts is that they are not context-sensitive enough to capture the intended meanings. They seem to excessively constrain what generics may be used to assert.

Nguyen (2020) does not claim that all presently existing accounts fail to accommodate the positive data. In particular, he concedes that Sterken's (2015a) indexical account can accommodate the positive data. Sterken (2015a) argues that a covert indexical in the logical form of generics can explain the positive data. On the other hand, Nguyen (2020) argues that we do not need to posit any covert structure to explain the positive data. He argues for a pragmatic, Neo-Gricean explanation that appeals to the varying communicative intentions of speakers. Both accounts are specifically developed to accommodate the rampant contextsensitivity of generics. It should then be no surprise that they can accommodate the positive data. 
For the purposes of this paper, we are content to simply assume that only Nguyen's (2020) and Sterken's (2015a) accounts can capture the positive data. We do not see this as significantly limiting the interest of our investigation here. In the next section, we will introduce new data and defend linguistic generalizations of broad semantic interest. Moreover, we will establish a conditional claim about which account is more preferable (when appropriately modified): If the pragmatic account and the indexical account are the only accounts that can accommodate the positive data, then the indexical account is best. As we endorse Nguyen's argument from the positive data against other accounts, we find the indexical account, at least when appropriately modified, the best view on generic meaning.

Before introducing some new data, let us describe the two accounts in some further detail. Among semanticists, it is generally accepted that there is a dyadic operator $G E N$ whose syntactic and semantic function is like an adverb of quantification (e.g., "usually" and "generally"). Fara (2006) argues that adverbial quantifiers can freely quantify over situations or individuals. For example, "Scandinavians usually have blue eyes" can have roughly the same reading as "most Scandinavians have blue eyes." For simplicity of exposition, we are going to accept Fara's conjecture and assume that GEN also can freely quantify over either individuals or situations. If GEN quantifies over situations, generic sentences have habitual readings (e.g., "Mary smokes," "Steve runs," etc.). If GEN quantifies over individuals, then they have individual (or I-generic) 
readings (e.g., "Barns are red," "Ravens are black," etc.). In this paper, we

focus on individual readings of generic sentences.

The main difference between Sterken (2015a) and Nguyen (2020)

lies in whether GEN is posited. Sterken (2015a) posits GEN. But on her

account, GEN is not any particular quantifier. Instead, it is an indexical

over quantifiers. There are broadly two ways to formulate this claim. One

is to say that the semantic value of GEN is determined by a Kaplanian

character (Kaplan 1989). The other is to say that its semantic value is not

determined but merely constrained by some semantic component (Cooper

1983; Heim and Kratzer 1998). ${ }^{6}$ Either way, her indexical theory is

flexible enough to capture the positive data. On her view, quantifiers can

range over merely possible situations as well as actual ones. Moreover,

\footnotetext{
${ }^{6}$ Kaplan (1989) distinguishes between pure indexicals (e.g., "I", "here", "now", etc.) and true demonstratives (e.g., "he", "she", "that", etc.). In the case of pure indexicals, the linguistic conventions that constitute the meaning of a pure indexical (= characters) determine the referent of an indexical in a given (public) context (e.g., the speaker, the time, the location of the utterance). For example, when one uses "I", the speaker automatically refers to herself. No pointing or intentions are required to determine its referent. But in the case of true demonstratives, the conventional meaning of a demonstrative does not automatically determine its referent in a given (public) context. Demonstratives require the speaker's demonstrations or intentions to fix their referent. The main difference between pure indexicals and true demonstratives is whether the conventional meaning of an expression automatically determine its referent in a given (public) context or not. This is what we wish to capture by distinguishing the Heim and Kratzer account from the Kaplanian account. On Kaplan's character-based account, the conventional meaning of a pronoun automatically determines its referent in a context. On Heim and Kratzer's account, the conventional meaning of a pronoun just constrains, but not determines, its referent.

Furthermore, there is a difference in formalizations between the two. On the Kaplanian account, the meaning of a pure indexical is a character, which is a function from (public) contexts to contents. On Heim and Kratzer's account, the meaning of a pronoun is defined in terms of a variable assignment function, and the variable assignment function is a function from indices to individuals. The variable assignment function represents the utterance context, but Heim and Kratzer's notion of context should be understood as including the speaker's intentions, so that it shouldn't be confused with Kaplan's narrow notion of context.
} 
GEN can in principle have any quantifier as its semantic value. Therefore, Sterken can handily accommodate the positive data. For example, Sterken's view captures the truth of (6) by allowing GEN to have the semantic value of a particular quantifier over ideal situations: This quantifier has the meaning of "all ideal."

On the other hand, according to Nguyen (2020), generic sentences fail to express propositions. He does not posit GEN. He leaves it open whether the logical form of "Fs are Gs" is just "[Fx][Gx]" or "Ux[Fx][Gx]," ${ }^{7}$ where $U$ is a syntactically real but semantically null operator. So, its quantificational force should be supplied by a pragmatic process (e.g., Bach's (1994) impliciture, Recanati's (2004) free enrichment, Sperber and Wilson's (1986) explicature). On Nguyen's view, speakers use generics to convey propositions built out of the nonpropositional semantic content of the uttered generic. Which proposition — built out of the non-propositional content—is conveyed depends on the speaker's communicative intentions. The speaker's communicative intentions are sensitive to the goal of the conversation and her beliefs about which propositions her audience will grasp. Here, to grasp a proposition is to correctly identify it as a proposition that the speaker intends to convey. Nguyen explains the positive data by allowing speakers' communicative intentions to vary significantly in different

\footnotetext{
${ }^{7}$ The former logical form is that of an open sentence. The latter logical form is that of a quantified sentence in which a variable is bound by the operator, $\mathrm{U}$, in the quantifier position.
} 
contexts. On Nguyen's view, a variety of communicative intentions leads to a variety of propositions asserted.

To summarize, the key difference between Sterken (2015a) and Nguyen (2020), then, is whether there is a semantically effective operator or not. Sterken posits GEN. Nguyen does not.

\section{The Negative Data and Its Implications}

Let's now turn our attention to some new data. In this paper, we present some new data - which we will call the Negative Data-and develop a more sophisticated version of the indexical account to explain the data. Our key observation is that there are some quantificational forces that generic utterances cannot convey. Consider the following data:

\section{The Negative Data ${ }^{8,9}$}

(1*) [\#Few] mosquitoes transmit malaria.

\footnotetext{
${ }^{8}$ The "\#" indicates that the depicted reading is unavailable. For example, $\left(1^{*}\right)$ reports that one cannot utter "mosquitoes transmit malaria" to non-figuratively mean that few mosquitos transmit malaria. Note that "few" and "a few" differ in meaning. "a few" is similar to "many" and it is upward monotone. "few" is similar to "not many" and it is downward monotone. See Barwise and Cooper (1981) for more details.

${ }^{9}$ Here are sample lexical entries for the quantifiers in the negative data:

【“"not all” $\rrbracket^{\mathrm{c}}=\lambda \mathrm{F} . \lambda \mathrm{G} . \frac{\#(\mathrm{~F} \& \mathrm{G})}{\# \mathrm{~F}}<1$

$\llbracket$ "not many" $\rrbracket^{\mathrm{c}}=\lambda \mathrm{F} \cdot \lambda \mathrm{G} \cdot \frac{\#(\mathrm{~F} \& \mathrm{G})}{\# \mathrm{~F}}<0.3$.

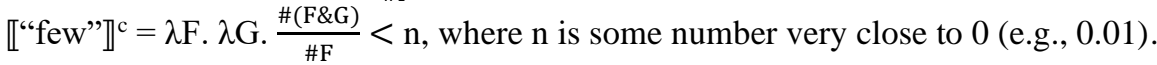

$\llbracket “ n o " \rrbracket c=\lambda F \cdot \lambda G \cdot \frac{\#(F \& G)}{\# F}=0$.

$\llbracket$ "“at most half" $\rrbracket^{c}=\lambda F \cdot \lambda G \cdot \frac{\#(F \& G)}{\# F} \leq 0.5$.

"“exactly five" $]]^{\mathrm{c}}=\lambda \mathrm{F} . \lambda \mathrm{G}$. \#(F\&G) $=5$.

$\llbracket$ "at least five" $\rrbracket^{c}=\lambda F . \lambda G$. \#(F\&G) $\geq 5$.
} 
$(2 *)$ [\#Not many] barns are red.

$\left(3^{*}\right)$ [\#Not all] prime numbers are odd.

(4*) [\#At most half of the] ravens [in the zoo] are black.

(5*) [\#By definition, no] squares are round.

(6*) [\#At least five] girls cry.

(7*) [\#Exactly five] orange crushers [can] crush oranges.

These quantificational readings are not available. Neither Sterken's (2015a) nor Nguyen's (2020) account fares well with the negative data. The negative data is evidence against the pragmatic approach. If the quantificational force of generics is pragmatically supplied, there seems to be no reason why they could not be enriched so as to make these readings available. There was a similar debate over the linguistic status of domain restriction between Recanati (2004) and Stanley (2007). Recanati (2004) accounts for domain restriction in terms of free enrichment but does not mention any pragmatic constraint on free enrichment. Stanley (2007, p.238) objects that Recanati's account then overgenerates readings. For example, "Every Frenchman is seated" can be used to express what "Every Frenchman in the classroom is seated" expresses (set intersection) but not what "Every Frenchman or Dutchman is seated" expresses (set union). Similarly, "Every Frenchman is seated" cannot be used to express the proposition that "Every non-Frenchman is seated" expresses (complement). Analogously, Nguyen's (2020) account may fare well with 
the positive data, but it seems unable to capture the negative data. This is a significant problem for his view.

Sterken's (2015a) account, as it is now, does not fare well with the negative data either. On Sterken's account, GEN is an indexical over quantifiers. But she doesn't specify what kind of indexical GEN is. As we can see from the negative data, GEN cannot take quantifiers like "no," "not all," "at least five," "at most half," "exactly five," etc as its value. But Sterken provides no good reason why GEN cannot take such quantifiers as its value.

In summary, given Nguyen's argument from the positive data against other accounts, only Sterken's indexical account and Nguyen's pragmatic account can explain the positive data. However, these two accounts still fail to explain the negative data. Thus, there is no available account on the market that can explain both the positive data and the negative data. In the remainder of this paper, we will develop a new version of the indexical account to accommodate those two sets of data. The basic strategy is that since GEN is semantically active, it may carry semantic features that rule out the quantificational forces in the negative data. More specifically, in Section 4, we argue that GEN carries the following two semantic constraints: upward monotonicity and nonsymmetry. In Section 5, we argue that the same strategy is not available for the pragmatic account. 


\section{The Semantic Features of GEN}

\subsection{Two generalizations}

As mentioned earlier, there are two approaches to indexicals and pronouns: Kaplanian characters (Kaplan, 1989) and semantic constraints (Cooper 1983; Heim \& Kratzer, 1998). We will adopt the second approach here. On this approach, just as the pronoun "she" comes with a semantic constraint that its semantic value be female, GEN may come with certain semantic constraints. Semantic constraints provide conditions that must be satisfied for an expression to be semantically interpretable (i.e., for it to have a defined semantic value).

Before investigating what semantic constraints GEN comes with, let us first explain the logical structure of quantified sentences and two important logical properties of quantifiers: monotonicity and symmetry. A quantifier is considered having a tripartite structure: (i) quantificational force, (ii) restrictor argument, and (iii) scope argument. The quantificational force specifies how strong a quantifier is. The restrictor argument specifies the domain over which the quantifier ranges. The scope argument specifies the property that is attributed to those members provided by the restrictor argument. For example, the logical form of the sentence "all tigers are striped" can be represented as "all x [tiger $(\mathrm{x})][$ striped $(\mathrm{x})]$," according to which the quantifier "all" takes "tigers" (i.e., the restrictor argument) and "be striped" (i.e., the scope argument) as its arguments. 
Here, we are going to confine our attention to the scope argument of a quantifier and define upward and downward monotonicity as follows: Given any scope argument A, the quantifier Q is upward monotone iff for any B and C such that B entails $C, Q(A)(B)$ entails $Q(A)(C)$. Q is downward monotone iff for any $B$ and $C$ such that B entails $C, Q(A)(C)$ entails $\mathrm{Q}(\mathrm{A})(\mathrm{B}) . \mathrm{Q}$ is monotone iff it is either upward or downward monotone. $\mathrm{Q}$ is non-monotone iff it is neither upward nor downward monotone.

To fix ideas, let us consider some examples. Some examples of upward monotone quantifiers are "some," "at least five," "a few," "many," 10 "most," and "all." Some examples of downward monotone quantifiers are "no," "not all," "not many," "few," "at most half," and "at most two." Finally, "exactly five" and "between two and seven" are nonmonotone quantifiers (Barwise and Cooper, 1981; Peters and Westerstahl, 2006; Westerstahl, 2019).

The other logical property that we are interested in is symmetry. A quantifier $\mathrm{Q}$ is symmetric iff for any $\mathrm{A}$ and $\mathrm{B}, \mathrm{Q}(\mathrm{A})(\mathrm{B})$ iff $\mathrm{Q}(\mathrm{B})(\mathrm{A})$. $\mathrm{Q}$ is non-symmetric iff there are some $A$ and $B$ such that $Q(A)(B)$ and $Q(B)(A)$ have distinct truth-values. Existential quantifiers such as "some," "at least five," "exactly five," and cardinal "many" are symmetric. On the other

\footnotetext{
${ }^{10}$ It is widely accepted that quantifiers like "many," "a few," "few" have ambiguous readings (i.e., cardinal and proportional readings) (Partee 1988). Both cardinal and proportional "many" are upward monotone.
} 
hand, "all," "most," and proportional "many" are non-symmetric (Barwise \& Cooper, 1981; Peters \& Westerstahl, 2006; Westerstahl, 2019).

To draw proper semantic constraints on GEN, let us make two generalizations that we draw from the positive and negative data. We will use these generalizations in order to infer which semantic constraints to impose on GEN:

Generalization 1: The quantifiers in the positive data (e.g., proportional "a few," proportional "many," "most," "almost all," "all”) are all upward monotone and non-symmetric.

Generalization 2: The quantifiers in the negative data (e.g., "few," "not many," "not all," "no," "at most half," "at least five," "exactly five") are either downward monotone, non-monotone, or symmetric.

Generalization 1 seems intuitively plausible from considering generic utterances in various contexts. It seems that the quantifiers involved in the positive data (e.g. "a few" and "all") are all upward monotone.

Furthermore, other approaches also entail that generics are upward monotone. For example, according to normalcy-based approaches (Asher \& Morreau, 1995; Pelletier \& Asher, 1997), generics of the form "Fs are Gs" are roughly analyzed as "all normal Fs are Gs." Also, on probabilistic accounts (Cohen, 1996, 1999, 2004a,b), "Fs are Gs" are roughly analyzed 
as "most Fs are Gs" or "many Fs are Gs." Since "all," "most," and "many" are upward monotone, several other approaches on generics are also committed to the upward monotonicity of generics.

One might object at this point that there seem to be counterexamples to Generalization 1 such as the following:

(8) a. Lions are on the lawn. (Existential Bare Plurals)

b. Auto mechanics are available.

(9) a. A: Birds lay eggs. (Existential Generics)

B: Mammals lay eggs too.

b. A: No Indians eat beef.

B: That's not true. Indians do eat beef!

(10) a. Dutchmen are good sailors. (Port Royal Examples)

b. $\nRightarrow$ Dutchmen are sailors.

(11) a. Bulgarians are good weightlifters.

b. $\nRightarrow$ Bulgarians are weightlifters.

(12) a. Bears are brown, black, and white. (Conjunctive Generics)

b. $\nRightarrow$ Bears are brown.

(13) a. Chickens lay eggs. (Alternative-Sensitivity)

b. $\nRightarrow$ Chickens are female.

(i) Existential Bare Plurals: If GEN is non-symmetric, generics shouldn't be able to have an existential quantificational force, because the 
existential quantifier "some" is a symmetric quantifier. But (8a) and (8b) seem to have existential readings. That is, (8a) can mean that some lions are on the lawn, and (8b) can mean that some auto mechanics are available. However, it is well-known that bare plurals are ambiguous between generics and existentials and thus, in addition to generic readings, they can at least sometimes have existential readings. If so, (8a) and (8b) express simple existential readings and pose no problem for a theory of generics. In this paper, we remain neutral on how existential bare plural are derived. ${ }^{11}$

(ii) Existential Generics: Even if examples like (8a) and (8b) are treated as simple existential bare plurals, Cohen (2004a) argues that there are true generics with an existential flavor. He argues that in (9a) and (9b), B's response expresses a generic reading with an existential force. B's response in (9a) has an existential force because it can be true even if platypuses and echidnas are the only egg-laying mammals. Also, B's response in (9a) has a generic reading because it expresses a law-like generalization that is not temporary but is expected to happen regularly or normally in the future. He says that even if no platypuses and echidnas happen to lay eggs this year and so no mammals lay eggs this year, the sentence "Mammals lay eggs too" would still be true, given that this is just a temporary situation and in the future they are expected to lay eggs normally. Similarly, he says that B's response in $(9 b)$ has a generic reading

${ }^{11}$ For references, see Carlson (1977), Diesing (1992), Kratzer (1995) and Chierchia (1998). 
because, to be true, the sentence "Indians do eat beef" requires some instances of Indians who regularly eat beef throughout a long period of time.

As we noted in Section 2, GEN may quantify over either individuals or situations. If GEN quantifies over individuals, generic sentences have individual readings (e.g., "Barns are red," "Ravens are black," etc.), and if GEN quantifies over situations, generic sentences have habitual readings (e.g., "Mary smokes," "Steve runs," etc.). We believe that existential generics are not individual-level generics but habituals. More specifically, we propose that existential generics arise when the existential quantifier mentioned in the discussion of (8) quantifies over individuals and GEN quantifies over situations. As a result, "Mammals lay eggs, too" has a reading similar to "Some mammals normally lay eggs," and "Indians do eat beef" has a reading closer to "Some Indians regularly eat beef." In those paraphrases, "some" represents the contribution of the existential (and non-generic) quantifier, and "normally" or "regularly" represent the contribution of GEN.

(iii) Port Royal Examples: If GEN is upward monotone, (10)-(13) should be valid. But those inferences seem invalid. Examples (10)-(13) can be broken down into three kinds. Let us consider them one by one. (10) and (11) are the classic Port Royal examples. They have traditionally been thought to be troublesome for semantic theories of generics. But Leslie (2008) argues that if we properly understand the logical forms of the Port 
Royal examples, they are not troublesome at all. Her solution draws its inspiration from Larson's (1998) proposal for non-generic variants of the Port Royal examples.

(14) a. Barry is a good dancer ( $\approx$ Barry's dancing is good).

b. $\nRightarrow$ Barry is a dancer.

Larson (1998) proposes that when an agentive nominal is modified by an adjective, ${ }^{12}$ the agentive nominal occurs as a part of the restrictor argument, not the scope argument. Leslie (2008) generalizes this solution to the generic versions of the Port Royal examples and proposes that "sailors" in (10) and "weightlifters" in (11) appear as part of the restrictor argument. If Leslie's (2008) proposal is right, the logical forms of (10a) and (11a) would be just like those of (15a) and (15b).

(15) a. Dutch sailors are good (qua sailors).

b. Bulgarian weightlifters are good (qua weightlifters).

But (15) does not threaten Generalization 1. Plausibly, being good at something entails being adequate at it. But note that (15a) does entail that

\footnotetext{
${ }^{12}$ We believe this phenomenon is not just restricted to agentive nominals or modification by adjectives. Consider the following example (Cohen 2001). (L) roughly means that most linguists who came did so by bus or that for most events $e$, if $e$ is a linguist's coming, $e$ is done by bus.

(L) Most linguists came by bus.
} 
Dutch sailors are adequate (qua sailors) and that (15b) does entail that Bulgarian weightlifters are adequate (qua weightlifters). Upward monotonicity is preserved.

(iv) Conjunctive Generics: Another issue concerns conjunction. "Bears are brown, black, and white" is felicitous, but "Bears are brown" is not. ${ }^{13}$ We believe that this contrast is due to a Quantity-1 implicaturewhich requires cooperative speakers to make their conversational contributions as informative as is required - in Grice's (1989) sense. Some evidence for this claim is that cancelation is felicitous: "Bears are brown, but of course they can also be black or white" is felicitous. This result suggests that "Bears are brown" is infelicitous only because an utterance of this sentence usually implicates, incorrectly, that bears cannot have other colors. To accommodate conjunctive generics like "Bears are brown, black, and white," we follow Leslie (2015) in allowing conjunction to take scope over GEN. In other words, "Bears are brown, black, and white" can mean "Bears are brown, bears are black, and bears are white."

(v) Alternative-Sensitivity: (13) is one of what Leslie (2008) classifies as troublesome generics. A common intuition is that "Chickens are female" is false even though "Chickens lay eggs" is true and all egglaying chickens are female. Cohen (2001) provides a natural explanation for this phenomenon. He argues that GEN is sensitive to contextually

\footnotetext{
${ }^{13}$ This latter datapoint is contestable. Some of our informants claim that "Bears are brown" is felicitous. If it is indeed felicitous, then of course our account can straightforwardly accommodate that.
} 
determined alternatives. This should not be surprising, since many proportional quantifiers like "many" and "a few" are evaluated relative to a set of alternatives. For example, (13a) may be true because it is evaluated relative to a set like \{ giving birth to live young $\}$. On the other hand, (13b) may be false because it is evaluated relative to a set like $\{$ being male\}. There can be many ways to implement alternative-sensitivity in a formal system. For example, alternative sets can be understood as sets of properties or sets of propositions. Also, we can compare a generic sentence with each alternative in the alternative set, the average of the alternative set, or the union of the alternative set, etc. We will return to the issue of how to implement alternative-sensitivity in Section 4.2.

But one might ask why we should think that GEN is, at least sometimes, evaluated with respect to alternatives. On the indexical approach, GEN is an indexical over quantifiers. If Generalization 1 is correct, GEN may denote proportional "a few" and proportional "many." Since proportional "a few" and proportional "many" are alternativesensitive (Cohen, 2001), GEN is alternative-sensitive as well when it denotes these quantifiers. And perhaps there are non-lexicalized, alternative-sensitive, upward monotone quantifiers that GEN can denote.

Furthermore, there is some independent, empirical data indicating that GEN is alternative-sensitive. Note that, as Cohen (2001) observed, proportional quantifiers and GEN are not conservative. A quantifier Q is conservative iff " $Q x[F(x)][G(x)]$ ” is always equivalent to " $Q x[F(x)][F(x)$ 
$\wedge \mathrm{G}(\mathrm{x})]$." For example, "some" is conservative, as evidenced by the equivalency of (16a) and (16b). On the other hand, "many" and GEN are not conservative, as evidenced, respectively, by the lack of equivalence between (17a) and (17b) and the lack of equivalence between (18a) and $(18 b) .{ }^{14}$

(16) a. Some politicians tell the truth.

b. Some politicians are politicians who tell the truth.

(17) a. Many Scandinavians have won the Nobel Prize in Literature.

b. Many Scandinavians are Scandinavians who have won the Nobel

Prize in Literature.

(18) a. Mosquitoes transmit malaria.

b. Mosquitoes are mosquitoes that transmit malaria.

(16a) and (16b) entail one another. This result indicates that "some" is conservative. On the other hand, (17a) does not entail (17b). After all, (17a) is true even though (17b) is false. ${ }^{15}$ So, "many" is not conservative. Finally,

${ }^{14}$ Cohen (2001) discusses (17) and agrees with our judgment that (17a) does not entail (17b).

${ }^{15}$ The Nobel Prize in Literature is an award for which authors of any nationality are eligible. As of 2019, 16 of the 116 recipients were Scandinavians. For some comparison: 11 were American, one was Chinese, and one was Indian. Given these facts, (17a) seems true to us. If you disagree, then you may imagine that all 116 previous recipients were Scandinavian. In such a scenario, (17a) is obviously true and (17b) is still false. 
(18a) does not entail (18b). After all, (18a) is true even though (18b) is false. ${ }^{16}$

So, GEN is not conservative. But this then indicates that GEN is evaluated with respect to contextually determined alternatives. Intuitively, conservative quantifiers only see objects that have the restrictor property. For example, the truth of "Some dogs bark" depends only on the behavior of dogs, not on the behavior of non-dogs. On the other hand, nonconservative quantifiers see objects that do not have the restrictor property. For example, the truth of (17a) can depend on how many nonScandinavians have won the Nobel Prize in Literature. ${ }^{17}$ Holding fixed the fact that 16 Scandinavians have won this award, it would make a difference to our assessment of (17a)'s truth whether only one nonScandinavian or over five thousand non-Scandinavians have won the award. But this attention to individuals that do not have the restrictor property indicates that non-conservative quantifiers are alternativesensitive. Since GEN is non-conservative, it follows that the truth-value of a generic "Fs G" can, at least sometimes, depend on the properties of non-Fs.

To summarize, there is an independent reason for why the inference from (13a) to (13b) sounds invalid. That is, (13a) and (13b)

\footnotetext{
${ }^{16}$ Less than $1 \%$ of mosquitoes transmit malaria. There are over 3,000 species of mosquitoes and only the females of one species are capable of transmitting malaria. Given these facts, we judge (18a) to be still be true even though (18b) is false.

${ }^{17}$ We ignore the absolute proportional reading of "many" here. This is appropriate as we are only interested in its relative proportional reading. To help aid us in focusing on the intended reading here, assume that 16 of 116 does not count as absolutely many Scandinavians.
} 
might have different truth-values because they are evaluated relative to different alternative sets. ${ }^{18}$ If this is the case, there is an implicit context change in the above inference from (13a) to (13b). If so, then (13a) and (13b) do not constitute a genuine counterexample to Generalization 1. That is, given that generics are at least sometimes alternative-sensitive, we can preserve the upward monotonicity of generics. The quantificational force of generic utterances is always upward monotone.

Upward monotonicity is sufficient to single out the quantifiers in the positive data (i.e., proportional "a few," proportional "many," "most," "almost all," "all"). So, one might question why we include non-symmetry in Generalization 1. We do so because non-symmetry is necessary to explain the negative data. Upward monotonicity explains why GEN cannot be used to express downward monotone quantifiers like "few," "not many," "not all," "no," "at most half" and non-monotone quantifiers like "exactly five" (Peters and Westerstahl 2006, Section 5.2.). But it doesn't explain why GEN cannot be used to express upward monotone cardinal quantifiers like "at least five". Non-symmetry can be used to exclude these quantifiers, because they are symmetric. For example, "at least five" is symmetric because "At least five $(\mathrm{x})[\mathrm{F}(\mathrm{x})][\mathrm{G}(\mathrm{x})]$ " is true iff

\footnotetext{
${ }^{18}$ We believe this generalizes to other seeming non-Port-Royal counterexamples to Generalization 1. For example, suppose that $1 \%$ of Frenchmen regularly eat horsemeat and that no Frenchmen eat any other kinds of meat. In such a situation, "Frenchmen eat horsemeat" seems true but "Frenchmen eat meat" seems false. This is so even though any Frenchman who eats horsemeat eats meat. Alternative sensitivity captures this data in a satisfying way. Given alternative sensitivity, we can say that "Frenchmen eat meat" is false since Frenchmen are so much less likely to eat meat than those of other nationalities. Moreover, we can say that "Frenchmen eat horsemeat" is true since Frenchmen are so much more likely to eat horsemeat than those of other nationalities.
} 
"At least five $(\mathrm{x})[\mathrm{G}(\mathrm{x})][\mathrm{F}(\mathrm{x})]$ is true. If at least five boys run, then at least five runners are boys - and vice-versa. Therefore, there is good reason to include non-symmetry in Generalization $1 .{ }^{19}$

Generalization 2 seems plausible upon reflection. Any instance of the negative data seems to involve a downward monotone, non-monotone, or symmetric quantifier. Our defense of Generalization 1 partially supports this generalization. But we can say more in defense of Generalization 2. We will use a three-pronged strategy. First, we will appeal to data about NPIs (negative polarity items) to show that generics do not involve downward monotone quantifiers. Second, we will appeal to data about inference patterns involving conjunctive predicates to show that generics do not involve non-monotone quantifiers. Third, we will appeal to data about inference patterns to show that generics do not involve symmetric quantifiers. Since Generalization 2 just says that generics do not involve downward monotone, non-monotone, or symmetric quantification, our three-pronged strategy directly supports Generalization 2.

\footnotetext{
${ }^{19}$ The negative data suggests that GEN cannot take the same quantificational forces as "few," "not many," "not all," "at most half," "no," "at least five," and "exactly five." "Few," "not many," "not all," "at most half," and "no" are downward monotone, and "exactly five" is non-monotone, so they are excluded by the upward monotonicity condition (Peters and Westerstahl 2006, Section 5.2.). "No," "at least five," and "exactly five" are symmetric, so they are excluded by the non-symmetry condition (Westerstahl 2019, Section 13). "No" and "exactly five" are excluded by either condition.
} 
Let us proceed. First, the hypothesis that generics cannot involve downward monotone quantifiers is empirically supported by the observation that they do not license NPIs like "at all" and "ever":

(19) \# Ravens are black at all.

(20) \# Chickens ever lay eggs.

The most popular account of how NPIs get licensed is the downward entailment account (Ladusaw, 1980). On this account, NPIs can only appear in downward entailing contexts. So, if GEN is downward entailing, (19) and (20) should be grammatically acceptable. But they are not. Observe the contrast with uncontroversially downward entailing quantifiers such as "no" and "few."

(21) No ravens are black at all.

(22) Few chickens ever lay eggs.

While this account of NPIs has recently been put into doubt, its main competitor, Giannakidou's (2002) non-veridicality account, also entails that downward entailing contexts license NPIs. Therefore, the distribution of NPIs in generics indicates that generics cannot involve downward monotone quantifiers. 
Next, we wish to point out some data on inference patterns involving conjunctive predicates that suggest generics cannot involve nonmonotone quantifiers. Observe that (23a) asymmetrically entails (23b), that (24b) asymmetrically entails (24a), and that neither (25a) nor (25b) entails the other:

(23) a. All writers are observant and creative.

b. All writers are observant.

(24) a. No writers are observant and creative.

b. No writers are observant.

(25) a. Exactly five writers are observant and creative.

b. Exactly five writers are observant.

(23a) entails (23b) because "all” is upward monotone. (24b) entails (24a) because "no" is downward monotone. Neither (25a) nor (25b) entails the other because "exactly five" is non-monotone. For our purposes here, it is most important to note that, if $\mathrm{Q}$ is a non-monotone quantifier, then neither “Q x $[F(x)][G(x)]$ ” nor "Q x $[F(x)][G(x) \wedge H(x)]$ ” entails the other. So if generics can sometimes involve non-monotone quantification, we should predict that generics sometimes pattern like (25). However, (26) and (27) show that this prediction does not hold: 
(26) a. Ravens are black and omnivorous. ${ }^{20}$

b. Ravens are black.

(27) a. Truth tables are tedious and boring.

b. Truth tables are tedious.

In fact, in each of (26) and (27), the (a)-sentence entails the corresponding (b)-sentence. So, if anything, generics pattern like (23), not (25). This result should not be surprising; Generalization 1 predicts this outcome..$^{21}$ Since generics do not pattern like sentences involving non-monotone quantification, we infer that generics do not involve non-monotone quantification.

But there is a seeming counterexample to the claim that generics do not involve non-monotone quantification. Consider (28):

(28) a. Frenchmen eat horsemeat.

b. Frenchmen eat meat.

It seems that neither (28a) nor (28b), strictly speaking, entails the other. To see that (28a) does not entail (28b), imagine a world just like ours except only $1 \%$ of Frenchmen ever eat horsemeat and this is the only sort

20 "Ravens are black and omnivorous" seems ambiguous between the following two: one in which GEN takes scope over conjunction and one in which conjunction takes scope over GEN. Here, we are interested in the first reading. On this reading, (26a) roughly means that for most $\mathrm{x}$, if $\mathrm{x}$ is a raven, $\mathrm{x}$ is black and omnivorous.

${ }^{21}$ (26) and (27) thus provide further evidence for Generalization 1. 
of meat that any Frenchmen ever eats. Even though the only meat any Frenchmen eat in our hypothetical scenario is horsemeat, it is still distinctive of Frenchmen that they eat horsemeat. Yet, it is relatively rare for a Frenchmen to eat meat at all. In such a scenario, (28a) is intuitively true and (28b) is intuitively false. To see that (28b) does not entail (28a) is easier. Imagine that every Frenchmen regularly eats meat—-say, beefbut that no Frenchman ever eats horsemeat. In such a scenario, (28b) is intuitively true and (28a) is intuitively false. So, neither (28a) nor (28b) entails the other. But then wouldn't this be evidence that generics can sometimes pattern like (25) and thus that it is possible for generics involve non-monotone quantification?

Our response to this purported counterexample will be in effect the same as our response to the purported counterexample that (13) posed to Generalization 1. We believe that alternative sensitivity is in play. Basically, (28a) and (28b) are evaluated with respect to different contexts. When evaluating (28a), we are intuitively comparing the proportion of Frenchmen who eat horsemeat against the proportion of Peruvians who eat horsemeat, the proportion of Koreans who eat horsemeat, the proportion of Ethiopians who eat horsemeat, etc. As it is relatively distinctive of Frenchmen that they eat horsemeat-even if only $1 \%$ of them eat horsemeat - this makes it easier for (28a) to be true than (28b) to be true. After all, when evaluating (28b), we are intuitively comparing the proportion of Frenchmen who eat meat against the proportion of Peruvians 
who eat meat, the proportion of Koreans who eat meat, the proportion of Ethiopians who eat meat, etc. We believe that if it were not for the distorting effects of alternative sensitivity, it would be transparent that (28a) entails (28b). But given the presence of alternative sensitivity, it is in practice very difficult to evaluate (28a) and (28b) with respect to the same context and thus the same set of alternatives. Therefore, (28) does not present a decisive objection to our claim that generics cannot involve non-monotone quantification. We will come back to the issue of how to implement this idea in a formal system in Section 4.2.

Lastly, we wish to point out data on inference patterns that suggest generics are not symmetric. A quantifier $Q$ is symmetric when $Q(A)(B)$ iff $\mathrm{Q}(\mathrm{B})(\mathrm{A})$. "Some" is a typical example of a symmetric quantifier. "Some student lives in Los Angeles" is truth-conditionally equivalent to "Someone who lives in Los Angeles is a student." However, (29)-(32) suggest that generics are not symmetric.

(29) a. Dogs are four-legged.

b. $\nRightarrow$ Four-legged animals are dogs.

(30) a. Lions are mammals.

b. $\nRightarrow$ Mammals are lions.

(31) a. Ducks lay eggs.

b. $\nRightarrow$ Animals that lay eggs are ducks.

(32) a. Bulgarians are good weightlifters. 
b. $\nRightarrow$ Good weightlifters are Bulgarians.

We conclude that both Generalization 1 and Generalization 2 are secure. In the next section, we discuss how to incorporate these generalizations into the formal meaning of GEN.

\subsection{The formal meaning of GEN}

Based on these two generalizations, we propose to modify the indexical approach in a way that accommodates both the positive and negative data. It is widely accepted that a lexical item carries a variety of phonological, syntactic, and semantic features (Chomsky and Halle 1968, Chomsky 1981). For example, the English pronoun "he" has features for person, gender, and number (also known as phi-features). These features can be described as follows: [person: 3], [gender: masculine], [number: singular]. These features function as constraints on the possible value of "he." That is, "he" succeeds in semantically denoting something only when its denotation is masculine, singular, and neither a speaker nor an addressee. On this view, the pronoun "he" can be defined as in (33) (Cooper 1983; Heim and Kratzer 1998).

(33) A Lexical Entry for Pronoun "He"

$\llbracket “ \mathrm{He}_{\mathrm{i}} \rrbracket^{\mathrm{g}}=$ defined iff $\mathrm{g}(\mathrm{i})$ is masculine, singular, and neither a speaker nor an addressee 
if defined, $g(\mathrm{i})$.

Here, $\mathrm{g}$ is a contextually determined assignment function that assigns semantic values to variables. From the above lexical entry, we see that "he" is defined only if its respective semantic constraints are satisfied.

We propose that just as the pronoun "he" carries person, gender, and number features, GEN carries features for monotonicity and symmetry. Monotonicity and symmetry have been considered important logical properties of quantifiers, because they have been used to explain puzzling linguistic phenomena. For example, symmetry was proposed as a feature of the quantifiers that are licensed in the so-called existential "there"-sentences (e.g., "There are some philosophers in the garden" is fine, but "There are most philosophers in the garden" is not) (Peters \& Westerstahl, 2006, Ch.6.3.). Also, monotonicity plays a crucial explanatory role for the distribution of negative polarity items (NPIs). That is, negative polarity items such as "ever" and "at all" require a downward monotone environment to be licensed (e.g., "No philosopher will ever come" is fine but "Some philosopher will ever come" is not) (Ladusaw, 1980; Peters \& Westerstahl, 2006, Ch.5). Thus, if GEN is an indexical over quantifiers, it seems not surprising that GEN carries some features regarding monotonicity and symmetry.

Let's now see how to implement the idea in the meaning of GEN. The monotonicity and symmetry features can be described thus: 
[monotonicity: upward], [symmetry:-]. The first can be read as "GEN has the value 'upward' for the feature [monotonicity]." The second can be read as "GEN has the value '-' for the feature [symmetry]." As phi-features function as constraints on the possible value of "he," monotonicity and symmetry features can function as constraints on the possible value of GEN. That is, GEN's semantic constraint requires that its semantic value be upward monotone and non-symmetric. On this view, GEN can be defined as in (34).

\section{(34) A Lexical Entry for GEN}

["GEN ${ }_{\mathrm{i}}{ }^{\prime \prime} \rrbracket^{\mathrm{g}}=$ defined iff $\mathrm{g}(\mathrm{i})$ is upward monotone and non-symmetric if defined, $g(i)$.

Here, $\mathrm{g}$ is a contextually determined assignment function that assigns a semantic value to an index. Both "he" and GEN are defined only if their respective semantic constraint is satisfied. If the constraint is satisfied, then the expressed semantic content is determined by g. The exact details do not concern us here, but in the case of "he," $\mathrm{g}$ (when defined) assigns some contextually salient single male individual to an index; in the case of GEN, g (when defined) assigns some contextually salient upward monotone, non-symmetric quantifier to an index. ${ }^{22}$

\footnotetext{
${ }^{22}$ For a discussion of a meta-semantic account of how GEN gets the different semantic values that it does in different contexts, see Sterken (2015a). Roughly, the account sketched there says that, in any context c, GEN has as its semantic value a quantifier that
} 
Before moving on, we'd like to mention two things. First, one might wonder how alternative sensitivity enters the picture. Our response is that we don't have to encode alternative sensitivity in the meaning of GEN. On the indexical approach, GEN is an indexical over quantifiers, and some quantifiers (e.g., "many," "a few," etc.) are alternative-sensitive (Cohen, 2001). If GEN denotes an alternative-sensitive quantifier, it will exhibit alternative-sensitivity. If it denotes an alternative-insensitive quantifier (e.g., "all," “almost all," etc.), it won't exhibit alternativesensitivity.

For example, suppose that the meaning of proportional "many" is given as follows:

(35) A Lexical Entry for Proportional "Many"

$\llbracket “$ Many” $\rrbracket^{c}=\lambda F<e, t>. \lambda G_{<e, t>} . \frac{\#(F \& G)}{\# F} \geq \theta_{c}$, where (i) $\theta_{c}$ is some contextually salient large number and (ii) if the contrast class $\operatorname{Cont}(\mathrm{c})$ is non-empty, $\theta_{\mathrm{c}}$ is significantly greater than the average proportion of the contrast class Cont(c).

Condition (ii) is borrowed from Kennedy $(1999,2007)$. According to the above lexical entry, the semantic value of proportional "many" is welldefined only when both presuppositions (i) and (ii) are satisfied. If both

both (i) the speaker intends to convey in $\mathrm{c}$ and that (ii) a reasonable audience would recognize that the speaker intends to convey in c. 
presuppositions are met, proportional "many" is upward monotone because if $G$ entails $H$, then $\frac{\#(F \& H)}{\# F} \geq \frac{\#(F \& G)}{\# F}$. So if $\frac{\#(F \& G)}{\# F}$ meets the threshold $\theta_{\mathrm{c}}, \frac{\#(\mathrm{~F} \& \mathrm{H})}{\# \mathrm{~F}}$ must meet this threshold too. For example, if "Many students in my class are from LA" is true, "Many students in my class are from California" is true as well. (35) correctly predicts this result.

Let's now return to generics and consider some concrete examples. (26) is a good example of upward monotonicity and (28) is a seeming counterexample to upward monotonicity.

(26) a. Ravens are black and omnivorous. b. $\Rightarrow$ Ravens are black.

(28) a. Frenchmen eat horsemeat.

b. $\nRightarrow$ Frenchmen eat meat.

(35) gives us an intuitively plausible explanation of the above examples. (28a) is true because the salient contrast class for (28a) is \{ Americans eat horsemeat, Canadians eat horsemeat, Germans eat horsemeat, etc. $\}$ while the contrast class for (28b) is \{Americans eat meat, Canadians eat meat, Germans eat meat, etc.\}. (28a) is true because the proportion of Frenchmen eating horsemeat over Frenchmen is significantly greater than the average proportion of its contrast class. On the other hand, $(28 \mathrm{~b})$ is not true because the proportion of Frenchmen eating meat over Frenchmen is not significantly greater than the average proportion of its contrast class. 
Since (28a) and (28b) are evaluated relative to different contrast classes, there is an implicit context shift between them. So, (28) doesn't constitute a counterexample to upward monotonicity.

On the other hand, (26) is valid. It is intuitive that (26) isn't evaluated with respect to contrast classes. This is because (26a) and (26b) would still be felicitous no matter how many raven-alternative creatures are black and omnivorous. For example, (26a) and (26b) would be true even in contexts in which the only contextually salient raven-alternative creatures are crows. When the contrast class Cont(c) is empty, Condition (ii) is trivially satisfied. As we mentioned earlier, when all presuppositions are satisfied, proportional "many" is upward monotone. Thus, it is not surprising that the inference from (26a) to (26b) is valid.

Second, we are open to the possibility that GEN could be subject to further constraints. One possible constraint is a constraint on GEN's modal forces. Examples like (4), (6), and (7) are naturally interpreted as carrying some modal force. Thus, one might suggest that GEN always comes with some modal force: actual, possible, normal, deontic, etc. On this view, (1)-(3) can be considered carrying actual force, (4) normal force, (6) deontic force, and (7) dispositional force. This constraint might be needed to explain the phenomenon that (36) can be used to express (37) but cannot be used to express (38) and (39):

(36) Ravens are black. 
(37) [All normal] ravens are black

(38) [\#All] ravens [in the zoo] are black.

(39) [\#All black] ravens are black.

While (37) expresses a modalized quantificational force, (38) and (39) do

not. This observation is also consistent with the common assumption that generics do not carry any domain restriction device. ${ }^{23}$ The constraint

discussed here can simply be added to the definition of GEN as follows: ${ }^{24}$

(40) $\llbracket “ " G E N_{i}$ "’ $\rrbracket^{g}=$ defined iff $g(i)$ is upward monotone, non-symmetric, and modalized

${ }^{23}$ Leslie (2007) argues that if generics allow for domain restriction, "Ducks don't lay eggs" and "Ducks are female" should be fine, because we should be able to restrict the domain to either just male or just female ducks. But they are not fine. So, it seems that generics do not allow for domain restriction.

${ }^{24} \mathrm{~A}$ reviewer asks if further constraints are necessary in order to prevent overgenerating true readings of generics. For example, nothing in (34) or (40) prevent "Prime numbers are odd" from being given a true reading. For example, if GEN were assigned to a quantifier (e.g., "most (actual)") whose force is weaker than that of universal quantification, our semantics predicts that "Prime numbers are odd" is true. But normally, this generic is infelicitous and false. In reply, we will make two points. First, Sterken (2015a) and Nguyen (2020) have shown that "Prime numbers are odd" can be felicitous in some contexts. For example, if a classmate had to guess whether an arbitrarily chosen prime number were odd but had no idea whether prime numbers were more likely to be odd or even, another classmate could felicitously utter "Prime numbers are odd" as a hint. Here, the quantificational force seems to be that of "most." So, in fact, a semantics that predicted "Prime numbers are odd" could never be true would objectionably undergenerate true readings. Second, this broad overgeneration worry can be applied not just to indexical accounts of generics like ours and Sterken's (2015a), but-as Sterken (2015a) points out - also to the best available accounts on quantifier domain restriction, pronoun resolution, and gradable adjectives (with respect to the selection of a particular comparison class). Somehow pragmatics, broadly understood, plays a pivotal role in resolving the relevant contextual parameters for all of these phenomena. Even though we have no general answer to the question of what value GEN is assigned in a particular context, we do not find this result problematic. The problem of systematizing how contextual parameters are resolved is a problem for many theorists working on various semantic topics. Resolving this problem is a task for future work. All we can say now is that, just as pronouns are interpreted to refer to contextually appropriate individuals, GEN is also interpreted to denote contextually appropriate quantifiers. 
if defined, $g(i)$.

\subsection{GEN as the default mode of generalization}

In this section, we present a new observation. That is, not only GEN but also some other pronoun has the upward monotonicity constraint. The discussion in this section might be somewhat speculative, but we believe that this section inspires optimism that it is principled and not ad hoc to constrain GEN to be upward monotone.

Let's start with our observation. According to Wellwood (2019), "much" is a pronoun for measure functions (i.e., a function from entities or events to degrees). For our purposes, it is important to note that the denotation of "much" is restricted to those that are upward monotone.

(41) Ann bought as much coffee as Bill. (volume/weight o, temperature x) (42) Ann ran as much as Bill did. (distance/duration o, speed $\mathrm{x}$ )

"Much coffee" can be used to express a measure by volume or weight but not by temperature. Similarly, "run ... much" can be used to express a comparison by distance or duration, but not by speed. Note that for two objects $\mathrm{x}, \mathrm{y}$, when $\mathrm{x}$ is a part of $\mathrm{y}$, the volume/weight of $\mathrm{x} \leq$ the volume/weight of $y$. But it doesn't always hold that the temperature of $\mathrm{x}$ $\leq$ the temperature of $y$. Similarly, for two events $e_{1}, e_{2}$, when $e_{1}$ is a part

of $\mathrm{e}_{2}$, the distance/duration of $\mathrm{e}_{1} \leq$ the distance/duration of $\mathrm{e}_{2}$. But there is 
no guarantee that the speed of $\mathrm{e}_{1} \leq$ the speed of $\mathrm{e}_{2}$. Degree semanticists have tried to capture this linguistic phenomenon by using a particular semantic constraint. That is, the semantic value of "much" is restricted to upward monotone measure functions (Nakanishi, 2004, 2007; Schwarzschild, 2002, 2006; Wellwood, 2019). To summarize, GEN and "much" are both a kind of pronoun and they carry the upward monotonicity constraint.

Is there a deeper explanation for why GEN and "much" come with the same semantic constraint? We might find an answer in Leslie's (2008) suggestion that generics express the default mode of generalization, which is innate to us. When there is a sentence with no explicit quantifier, GEN is supplied by default. However, she doesn't provide any formal explanation of what this default mode of generalization is. ${ }^{25} \mathrm{We}$ can accept Leslie's (2008) claim that generics express our default mode of generalization. Our novel suggestion is that GEN does not express a particular kind of generalization but covers a certain range of generalizations - upward monotone, non-symmetric generalizations. So, assuming that GEN and "much" are both a sort of pronoun, it seems that

\footnotetext{
${ }^{25}$ What we mean by this claim is that Leslie does not offer an explanatory formal semantics for GEN. In both Leslie (2007) and Leslie (2008), she gives a merely disquotational semantics for GEN. On this view, the logical form of a generic "Fs G" just is "GEN $x[F(x)][G(x)]$," and there is nothing more for formal semantics to say on the issue. However, Leslie (2007) and Leslie (2008) do offer metaphysical truth conditions for the truth of a generic. But not only are these metaphysical truth conditions not semantically derived, but we also agree with Sterken's (2015b) objections to Leslie's view. Not only are there counterexamples to Leslie's proposed metaphysical truth conditions, but a semantics for generics should also predict that generics are contextsensitive. Leslie's disquotational semantics does not satisfy this latter condition. Thanks to an anonymous reviewer for encouraging us to discuss these issues.
} 
the above two seemingly independent phenomena can be explained by the same mechanism if upward monotonicity is constitutive of our default mode of generalization. ${ }^{26}$

\section{A Reply from Pragmatic Constraints?}

In the previous section, we have developed a novel version of the indexical view that accommodates both the positive data and the negative data. One might wonder if the same strategy can be applied to the pragmatic approach. Just as the indexical approach can accommodate the negative data by positing the right semantic constraint on GEN, proponents of the pragmatic view might claim that the pragmatic view can be saved if we posit the right pragmatic constraints on free enrichment. ${ }^{27}$

Many pragmatic theorists (e.g., Carston, 2004; Hall 2014; Recanati, 2004; Sperber \& Wilson, 1986) claim that there should be some pragmatic constraints on pragmatic processes like free enrichment. But there is a problem: They argue for the existence of pragmatic constraints but do not—when they do specify any at all—specify plausible and generalizable

\footnotetext{
${ }^{26}$ It is noteworthy that upward monotone quantifiers seem cognitively prior to downward monotone or non-monotone quantifiers. There is empirical evidence for this claim. Keenan and Westerstahl (2011) propose that all lexicalized quantifiers are monotone (whether upward or downward) and that almost all of them are upward monotone. Katsos et al. (2016) performed a cross-linguistic study suggesting that five-year-old children understand upward monotone quantifiers better than they do downward monotone quantifiers. Steinert-Threlkeld and Szymanik (2019) have developed a short-term memory neural network that learned upward monotone quantifiers more quickly than non-monotone quantifiers.

${ }^{27}$ Free enrichment, or pragmatic enrichment, is not free in the sense that anything goes. As Carston puts it, free enrichment is "the process of recovering unarticulated constituents... where what the process is 'free' from is linguistic control; obviously, it is tightly constrained by the pragmatic principles involved" (Carston 2004, pp. 818-819).
} 
constraints. After all, we need to be confident that these pragmatic constraints help explain both the positive and negative data even if the semantic contents of generics are mere propositional radicals in need of pragmatic enrichment. Proponents of the pragmatic approach need pragmatic constraints that do very specific work. For example, Nguyen (2020) uses Gricean conversational maxims to explain the positive data. But the negative data cannot be so easily explained. Suppose it is both true and conversationally relevant that not many mosquitoes transmit malaria. Nonetheless, the content of "Mosquitoes transmit malaria" cannot be pragmatically enriched so as to (non-figuratively) assert that not many mosquitoes transmit malaria.

Another example is Sperber and Wilson's (1986) relevance theory, on which pragmatic enrichments are allowed when they are sufficiently relevant. Here, relevance is a technical notion and is a function of positive cognitive effects (e.g., informativity) and additional cognitive processing burden. Additional positive cognitive effects lead to more relevance; additional cognitive processing cost detracts from relevance. Relevance theory claims that irrelevant candidate enrichments would be infelicitous, whereas sufficiently relevant ones would be felicitous. However, it is unclear why enriching generics with non-symmetric, upward monotone quantifiers would often lead to relevance and, on the other hand, enriching generics with symmetric, downward monotone, or non-monotone 
quantifiers would always lead to irrelevance. ${ }^{28}$ So, on relevance theory, the combination of the positive data and the negative data seem inexplicable on the pragmatic approach. We know of no viable pragmatic theory that will lead to a different result. The burden is on proponents of the pragmatic view to provide pragmatic principles that explain the data presented so far. If they are unable to do so, then they should adopt the indexical approach defended here.

\section{Conclusion}

We have presented some new data - the negative data - on generics and discussed its implication on the debate. Assuming Nguyen's contention that only the indexical account and the pragmatic account can accommodate the positive data, we argued that the indexical account is the only extant view that can explain both the positive data and the negative data. In this paper, we have developed and defended an improved variant of Sterken's indexical account. We do not deny that there might be other accounts that succeed in accommodating both data. But examining all possible accounts of generics is beyond the scope of this paper. What we hope to have established is that, in the absence of any viable alternative explanation of both the positive data and the negative data, we should

\footnotetext{
${ }^{28}$ In footnote 26, we mentioned that upward monotone quantifiers seem cognitively prior to downward monotone or non-monotone quantifiers. However, even if upward monotone quantifiers are cognitively prior to other quantifiers and thus likely less cognitively burdensome to process, why should only enrichments involving upward monotone quantifiers be relevant? After all, on relevance theory, any cost in cognitive processing could be outweighed by a high enough benefit in informativeness (or some other positive cognitive effect).
} 
accept an appropriately modified version of the indexical account. At the very least, the negative data puts pressure on the main contenders in the debate to explain why generic sentences do not have certain interpretations. Any empirically adequate theory on generics would need to be flexible enough to accommodate the positive data and yet constrained enough to accommodate the negative data. ${ }^{29}$

\section{References}

Asher, N., \& Morreau, M. (1995). What some generic sentences mean. In G. Carlson, \& F.J. Pelletier (Eds.), The generic book (pp. 300-339). Chicago University Press.

Bach, K. (1994). Conversational impliciture. Mind and Language 9(2), 124-162. https://doi.org/10.1111/j.1468-0017.1994.tb00220.x

Bach, K. (2002). Seemingly semantic intuitions. In J. Campbell, M. O'Rourke, \& D. Shier (Eds.), Meaning and truth: Investigations in philosophical semantics (pp. 21-33). Seven Bridges Press.

Bach, K. (2005). Context ex machina. In Z.G. Szabo (Ed.), Semantics versus pragmatics (pp. 15-44). Oxford University Press.

Barwise, J., \& Cooper, R. (1981). Generalized quantifiers and natural language. Linguistics and Philosophy 4(2), 159-219. https://doi.org/10.1007/BF00350139

\footnotetext{
${ }^{29}$ For helpful comments on earlier drafts of this paper, we wish to thank Mark Schroeder, Barry Schein, and Alexis Wellwood. For helpful comments during the review process, we wish to thank two anonymous reviewers from Philosophical Studies.
} 
Carston, R. (2004). Explicature and semantics. In S. Davis, \& B. Gillon (Eds.), Semantics: A reader (pp. 817-845). Oxford University Press.

Carlson, G. (1977). Reference to kinds in English. Ph.D. Dissertation. University of Massachusetts, Amherst.

Chierchia, G. (1998). Reference to kinds across languages. Natural Language $\quad$ Semantics $\quad$ 339-405. https://doi.org/10.1023/A:1008324218506

Chomsky, N., \& Halle, M. (1968). The sound pattern of English. Harper and Row.

Chomsky, N. (1981). Lectures on Government and Binding. Foris.

Cohen, A. (1996). Think generic: The meaning and use of generic sentences. Ph.D. Dissertation, Carnegie Mellon University.

Cohen, A. (1999). Generics, frequency adverbs and probability. Linguistics and Philosophy 22(3), 221-253. https://doi.org/10.1023/A:1005497727784

Cohen, A. (2001). Relative readings of many, often, and generics. Natural Language Semantics 9(1), 41-67. https://www.jstor.org/stable/23753090

Cohen, A. (2004a). Existential generics. Linguistics and Philosophy 27, 137-168. https://doi.org/10.1023/B:LING.0000016441.89129.3d 
Cohen, A. (2004b). Generics and mental representation. Linguistics and Philosophy $\quad$ 27, 529-556. https://doi.org/10.1023/B:LING.0000033851.25870.3e

Cooper, R. (1983). Quantification and syntactic theory. Reidel.

Diesing, M. (1992). Indefinites. MIT Press.

Fara, D.G. (2006). Descriptions with adverbs of quantification. Philosophical Issues 16, 65-87. https://doi.org/10.1111/j.1533$\underline{6077.2006 .00103 . x}$

Giannakidou, A. (2002). Licensing and sensitivity in polarity items: From downward entailment to (non)veridicality. In M. Andronis, A. Pycha, \& K. Yoshimura (Eds.), Papers from the 38th Annual Meeting of the Chicago Linguistic Society, Parasession on Polarity and Negation, CLS 38(2), 29-54. The University of Chicago.

Grice, H.P. (1989). Logic and conversation. In H.P. Grice (Ed.), Studies in the way of words (pp. 22-40). Harvard University Press.

Hall, A. (2014). 'Free' enrichment and the nature of pragmatic constraints. International Review of Pragmatics 6(1), 1-28. https://doi.org/10.1163/18773109-00601002

Heim, I. \& Kratzer, A. (1998). Semantics in generative grammar. Blackwell. 
Kaplan, D. (1989). Demonstratives. In J. Almog, J. Perry, \& H. Wettestein (Eds.), Themes from Kaplan, (pp. 481-563). Oxford University Press.

Katsos, N., Cummins, C., Ezeizabarrena, M., Gavarród, A., Kraljević, J. K., Hrzica, G., Grohmann, K. K., et al. (2016). Cross-linguistic patterns in the acquisition of quantifiers. Proceedings in the National Academy of Sciences, 113 (33), 9244-9249. https://doi.org/10.1073/pnas.1601341113

Keenan, E., \& D. Westerstahl. (2011). Generalized quantifiers in linguistics and logic. In J. van Benthem, \& A. ter Meulen (Eds.), Handbook of logic and language (2 $2^{\text {nd }}$ ed., 859-910). Elsevier.

Kennedy, C. (1999). Projecting the adjective: The syntax and semantics of gradability and comparison. Garland.

Kennedy, C. (2007). Vagueness and grammar: The semantics of relative and absolute gradable adjectives. Linguistics and Philosophy 30(1), 1-45. https://doi.org/10.1007/s10988-006-9008-0

Krifka, M., Pelletier, F., Carlson, G. ter Meulen, A., Chierchia, G., \& Link, G. (1995). Genericity: An Introduction. In G. Carlson and F. Pelletier (Eds.), The generic book (pp. 1-124). University of Chicago Press.

Kratzer, A. (1995). Stage-level and individual-level predicates. In G. Carlson and F. Pelletier (Eds.), The generic book (pp. 125-175). University of Chicago Press. 
Ladusaw, W. (1980). Polarity sensitivity as inherent scope relations. Garland.

Leslie, S. J. (2007). Generics and the structure of the mind. Philosophical Perspectives 21(1), 375-403. https://doi.org/10.1111/j.15208583.2007.00138.x

Leslie, S. J. (2008). Generics: cognition and acquisition. The Philosophical Review 117(1), 1-47. https://doi.org/10.1215/00318108-2007-023

Leslie, S.J. (2015). Generics Oversimplified. Noûs 49(1), $28-54$. https://doi.org/10.1111/nous.12039

Leslie, S. J. (2016, April 24). Generic generalizations. The Stanford Encyclopedia of Philosophy. https://plato.stanford.edu/entries/generics/

Nakanishi, K. (2004). On comparative quantification in the verbal domain. In Robert Young (Ed.), Proceedings of SALT XIV (pp. 179-196). CLC Publications.

Nakanishi, K. (2007). Measurement in the nominal and verbal domains. Linguistics and Philosophy 30, 235-276. https://doi.org/10.1007/s10988-007-9016-8

Nguyen, A. (2020). The radical account of bare plural generics. Philosophical $\quad$ Studies $\quad 177: \quad 1303-1331$. https://doi.org/10.1007/s11098-019-01254-8 
Partee, B. (1988). Many quantifiers. In J. Powers, \& K. de Jong (Eds.), Proceedings of the Fifth Eastern States Conference on Linguistics (pp. 383-402). Ohio State University.

Pelletier, F.J., \& N. Asher. (1997). Generics and defaults. In J. van Benthem, \& A. ter Meulen (Eds.), Handbook of logic and language (pp. 1125-1179). MIT Press.

Peters, S., \& D. Westerstahl. (2006). Quantifiers in language and logic. Clarendon Press.

Recanati, F. (2004). Literal Meaning. Cambridge University Press.

Schwarzschild, R. (2002). The grammar of measurement. In B. Jackson (Ed.), Proceedings of SALT XII (pp. 225-245). CLC Publications.

Schwarzschild, R. (2006). The role of dimensions in the syntax of noun phrases. Syntax 9(1), 67-110. https://doi.org/10.1111/j.14679612.2006.00083.x

Sperber, D., \& Wilson, D. (1986). Relevance. Blackwell.

Stanley, J. 2007. Language in Context (pp. 221-254). Oxford University Press.

Steinert-Threlkeld, S., \& Syzmanik, J. (2019). Learnability and semantic universals. Semantics \& Pragmatics 12(4), 1-35. I: http://dx.doi.org/10.3765/sp.12.4

Sterken, R. 2015a. Generics in context. Philosophers' Imprint 15(21), 130. http://hdl.handle.net/2027/spo.3521354.0015.021 
Sterken, R. 2015b. Leslie on generics. Philosophical Studies 172, 24932512. https://doi.org/10.1007/s11098-014-0429-2

Sterken, R. 2017. The meaning of generics. Philosophy Compass 12(8), 1-13. https://doi.org/10.1111/phc3.12431

Wellwood, A. (2019). The meaning of "more." Oxford University Press.

Westerstahl, D. (2019, July 26). Generalized quantifiers. The Stanford Encyclopedia of Philosophy. https://plato.stanford.edu/entries/generalized-quantifiers/ 